T. A. Hillier · A. Rousseau $\cdot$ C. Lange $\cdot$ P. Lépinay $\cdot$

M. Cailleau • M. Novak • E. Calliez •

P. Ducimetière $\cdot$ B. Balkau

\title{
Practical way to assess metabolic syndrome using a continuous score obtained from principal components analysis
}

\section{The D.E.S.I.R. Cohort*}

Received: 12 December 2005 / Accepted: 20 March 2006 / Published online: 16 May 2006

(C) Springer-Verlag 2006

\begin{abstract}
Aims/hypothesis: We devised a practical continuous score to assess the metabolic syndrome, and assessed whether this syndrome score predicts incident diabetes and cardiovascular disease. Subjects and methods: Among 5,024 participants of the Data from an Epidemiological Study on the Insulin Resistance Syndrome (D.E.S.I.R.)
\end{abstract}

*For details on the D.E.S.I.R. study, see Acknowledgements.

T. A. Hillier · A. Rousseau $\cdot$ C. Lange $\cdot$

P. Ducimetière $\cdot$ B. Balkau $(\triangle)$

National Health and Medical Research Institute (INSERM),

U258-IFR69, 16 Avenue Paul Vaillant Couturier,

94807 Villejuif cedex, France

e-mail: balkau@vjf.inserm.fr

Tel.: +33-1-45595161

Fax: $+33-1-47269454$

T. A. Hillier · A. Rousseau $\cdot$ C. Lange .

P. Ducimetière · B. Balkau

University Paris South, Faculty of Medicine,

Villejuif, France

T. A. Hillier

Center for Health Research Northwest/Hawaii,

Kaiser Permanente,

Portland, OR, USA

P. Lépinay

Health Examination Centre,

Orléans, France

M. Cailleau

Inter Regional Health Institute (IRSA), D.E.S.I.R. Study,

La Riche, France

M. Novak

IRSA, Health Examination Centre,

Chateauroux, France

E. Calliez

IRSA, Health Examination Centre,

Angers, France cohort, we defined a metabolic syndrome score by the first principal component ( $\mathrm{PC1}$ ), using only the correlations between continuous metabolic syndrome measures (glucose, waist circumference, triglycerides, and systolic blood pressure). This metabolic syndrome score was highly correlated with a similar score also including insulin and HDL cholesterol $\left(r_{s}=0.94\right)$. Over 9 years of follow-up, incident diabetes and cardiovascular disease (CVD) were predicted by logistic regression using the simpler metabolic syndrome score. Results: The means of the metabolic syndrome measures differed between men and women. Nevertheless, as the degree of variance explained and the PC1 coefficients were remarkably similar, we used a common metabolic syndrome score. The metabolic syndrome score explained $50 \%$ of the variance of the metabolic syndrome measures, and waist circumference had the highest correlation (0.59) with this score. Each standard deviation increase in the metabolic syndrome score was associated with a markedly increased age-adjusted risk of developing diabetes (odds ratios: men 3.4 [95\% CI 2.6-4.4]; women 5.1 [3.6-7.2]) and with increased incident CVD of 1.7 (1.4-2.1) in men and 1.7 (1.0-2.7) in women. Conclusions/interpretation: Our results, which should be confirmed in other populations, suggest that it is possible to evaluate the risk of the metabolic syndrome in a pragmatic fashion with a continuous score, obtained from principal components analysis of the basic, continuous syndrome measures.

Keywords Cardiovascular disease $\cdot$ Incidence · Metabolic syndrome - Principal components analysis . Syndrome X · Type 2 diabetes mellitus

Abbreviations ADA: American Diabetes Association . CVD: cardiovascular disease .

D.E.S.I.R.: Data from an Epidemiological Study on the Insulin Resistance Syndrome .

EASD: European Association for the Study of Diabetes .

NCEP: National Cholesterol Education Program .

OR: odds ratio $\cdot \mathrm{PC} 1$ : first principal component 


\section{Introduction}

The core components of the metabolic syndrome are now well known [1-3]. As defined by several expert committees [4-8], the metabolic syndrome (which includes hyperinsulinaemia and/or hyperglycaemia, central adiposity, hypertriglyceridaemia and/or hypo-HDL cholesterolaemia, and raised arterial pressure) predicts incident type 2 diabetes and cardiovascular disease (review, see [9]). Despite a plethora of studies on the metabolic syndrome, there is little research on a practical method to summarise the syndrome on the basis of a continuous score. Indeed, the recent joint American Diabetes Association-European Association for the Study of Diabetes (ADA-EASD) statement [10] issued an urgent call for necessary research on the metabolic syndrome, including research evaluating "A definition of the syndrome... that uses continuous variables in a multivariate score system".

The metabolic syndrome definitions in current use all count the number of measures that exceed certain thresholds, and the syndrome is considered present when the requisite number is met [4-8]. Although this counting allows all of these correlated measures to be considered, it implies that they all contribute equal risk. Furthermore, as for the syndrome itself, the risk associated with each measure is more likely to be continuous than dichotomous. Because of this dichotomisation, up to one-third of subjects initially classified as having the syndrome may be considered 'normal' 3 years later [11], despite minimal changes in the actual measures and presumed actual risk. The challenge, therefore, is to find the best method to summarise these highly correlated measures that comprise the syndrome as one continuous variable, and in a manner that is practical to use.

Principal components analysis is ideally suited to provide a continuous score of the syndrome, as the first principal component is, by definition, the linear sum of the measures that has the maximum possible variance. This contrasts with factor analysis, which would aim to determine the underlying structure of the syndrome $[12,13]$ by identifying fewer latent (unknown) factors. While a number of studies have used factor analysis to describe the metabolic syndrome [14 20], and some have used the first component or factor as a score [17-20], to our knowledge no studies have used an unrotated first principal component to provide a score that could be identified as the syndrome.

The aims of this study, therefore, were: (1) to extract the first principal component from the correlation matrix of the measures, the coefficients in this linear sum of measures being the correlations between the measures and the principal component; (2) to determine whether this continuous metabolic syndrome score is associated with incident diabetes and cardiovascular events after 9 years of follow-up; and (3) to illustrate with a nomogram how such a continuous metabolic syndrome score could be used simply to evaluate the metabolic syndrome in individuals.

\section{Subjects and methods}

\section{Study population}

The Data from an Epidemiological Study on the Insulin Resistance Syndrome (D.E.S.I.R.) study is a longitudinal cohort study that included 5,212 adults aged 30-65 years with the primary aim of describing the natural history of the insulin resistance syndrome. Subjects were recruited between 1994 and 1996 from volunteers insured by the French national Social Security system, which offers periodic health examinations free of charge. As part of the design of the cohort, men and women were recruited equally among 5-year age groups. Participants came from ten different Social Security Health Examination centres in western, central France. All subjects gave written informed consent, and the study protocol was approved by the Committee for the Protection of Human Subjects in Biomedical Research of Hôpital Bicêtre (Paris, France).

The metabolic syndrome was evaluated using the first principal component in the 5,024 adults in the D.E.S.I.R. cohort who had fasting blood samples and the following other core components of the metabolic syndrome measured at the baseline exam: glucose, insulin, triglycerides, HDL cholesterol, waist circumference, and blood pressure.

\section{Biological, anthropometric, and clinical measurements}

Venous blood samples were collected after a 12-h fast. Serum insulin was quantified by micro particle enzyme immunoassay with an automated analyser (IMX; Abbott, Rungis, France), and plasma glucose was assessed by an enzymatic method (modified glucose oxidase peroxidase) and automated analysers (Technicon RA 1000; Bayer Diagnostics, Puteaux, France; or Specific or Delta; Konelab, Evry, France). Serum HDL cholesterol and serum triglycerides were assayed, respectively, by the phosphotungstic precipitation and enzymatic Trinder methods, using an automated analyser (Technicon DAX24; Bayer Diagnostics; or Specific or Delta; Konelab).

A nurse or doctor measured height with a stadiometer (without shoes), weight (in light clothes), waist circumference with a tape measure (the smallest circumference between lower ribs and iliac crests), and systolic and diastolic blood pressure at rest (at least $5 \mathrm{~min}$ ) in a supine position on the right arm using a mercury sphygmomanometer.

The D.E.S.I.R. cohort was followed annually by questionnaire for updated medical information including new medical diagnoses and medication use. Follow-up exams were conducted every 3 years, with measurements performed by the same standardised protocol and methods, including laboratory processing. The year 9 exam occurred over a 16-month period, a mean of 8.5 years after the baseline exam (range 8-10 years). Diabetes was defined by a fasting plasma glucose $\geq 7.0 \mathrm{mmol} / 1$ or hypoglycaemic 
drug treatment. CHD, cerebrovascular disease, and peripheral vascular disease were physician-adjudicated by medical record review in participants who, on the follow-up questionnaires, reported new cardiovascular disease, chest pain, stroke, or leg pain when walking. CHD was only considered present if there was: (1) a documented myocardial infarction; (2) evidence of revascularisation by angioplasty, cardiac stent, or coronary artery bypass surgery; or (3) evidence of CHD (with medical management) based on abnormal exercise treadmill test, nuclear scintigraphy, or angiogram. For cerebrovascular disease, haemorrhagic infarcts were only included if they were clearly associated with hypertension or ischaemic disease. Peripheral vascular disease was only considered present if peripheral revascularisation was performed. Incident cardiovascular disease (CVD) was defined as development of CHD, cerebrovascular disease, or peripheral vascular disease during follow-up.

\section{Statistical methods}

All statistical analyses were performed with the SAS V8 System (SAS Institute, Cary, NC, USA). Baseline characteristics, means and percentages, were compared between men and women, using $t$ - and $\chi^{2}$ tests, with the Fisher exact test where necessary.

Principal components analysis enables the study of intercorrelated measures $[12,13]$ and uses either the observed variance-covariance or correlation matrix of the measures; the analysis produces linear combinations of the measures, the principal components. By definition, the first principal component (PC1) is the linear sum of the measures that has the largest total variance.

We studied the core measures of the syndrome with principal components analysis (fasting insulin, fasting glucose, waist circumference, triglycerides, HDL cholesterol, and systolic blood pressure) and their correlation

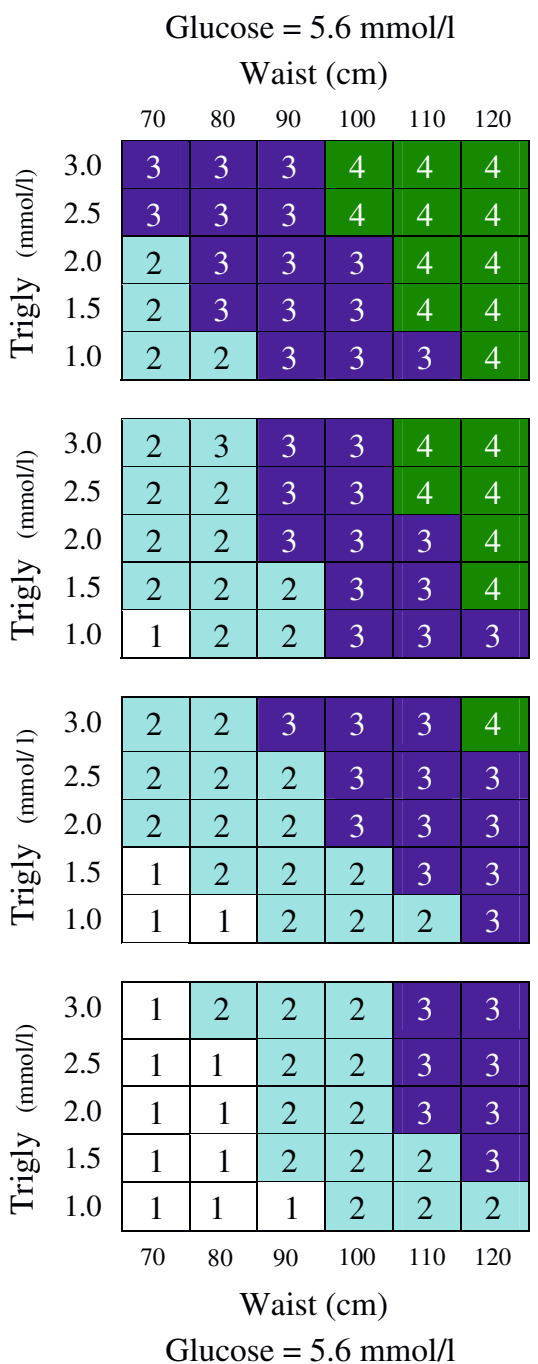

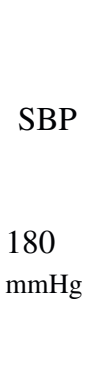

Glucose $=6.1 \mathrm{mmol} / \mathrm{l}$

$$
\text { Waist }(\mathrm{cm})
$$

\begin{tabular}{|c|c|c|c|c|c|}
\hline 70 & 80 & 90 & 100 & 110 & 120 \\
\hline 3 & 3 & 4 & 4 & 4 & 5 \\
\hline 3 & 3 & 3 & 4 & 4 & 4 \\
\hline 3 & 3 & 3 & 4 & 4 & 4 \\
\hline 2 & 3 & 3 & 3 & 4 & 4 \\
\hline 2 & 2 & 3 & 3 & 3 & 4 \\
\hline
\end{tabular}

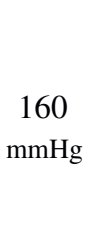

140
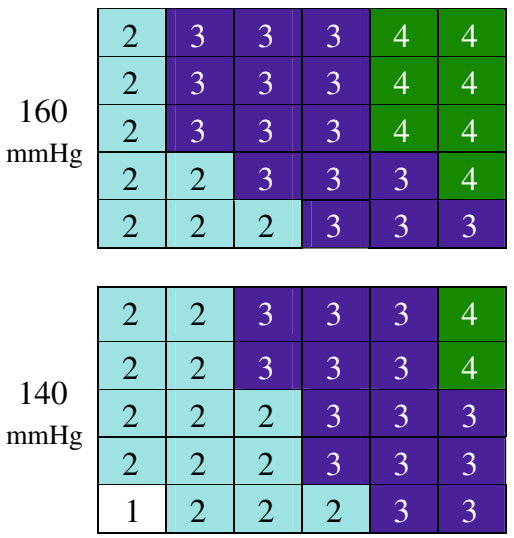

160

$\mathrm{mmHg}$
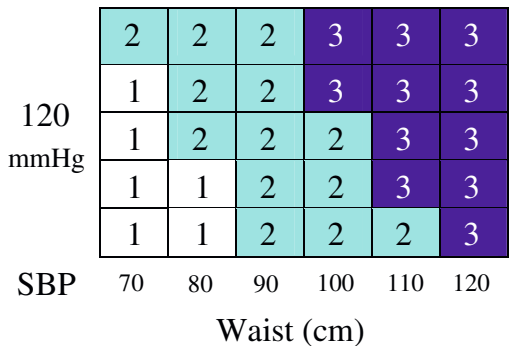

Glucose $=6.1 \mathrm{mmol} / \mathrm{l}$

140

120
Glucose $=7.0 \mathrm{mmol} / 1$

$$
\text { Waist }(\mathrm{cm})
$$

\begin{tabular}{|c|c|c|c|c|c|c|c|}
\hline SBP & 70 & 80 & 90 & 100 & 110 & 120 & \\
\hline & 3 & 4 & 4 & 4 & 5 & 5 & 3.0 \\
\hline & 3 & 3 & 4 & 4 & 4 & 5 & 2.5 \\
\hline & 3 & 3 & 4 & 4 & 4 & 5 & 2.0 \\
\hline & 3 & 3 & 3 & 4 & 4 & 4 & 1.5 \\
\hline & 2 & 3 & 3 & 3 & 4 & 4 & 1.0 \\
\hline
\end{tabular}

\begin{tabular}{|c|c|c|c|c|c|c|c|}
\hline \multirow{5}{*}{$\begin{array}{c}160 \\
\mathrm{mmHg}\end{array}$} & 3 & 3 & 3 & 4 & 4 & 5 & 0 \\
\hline & 3 & 3 & 3 & 4 & 4 & 4 & .5 \\
\hline & 2 & 3 & 3 & 4 & 4 & 4 & 2.0 \\
\hline & 2 & 3 & 3 & 3 & 4 & 4 & 5 \\
\hline & 2 & 2 & 3 & 3 & 3 & 4 & \\
\hline
\end{tabular}

\begin{tabular}{|c|c|c|c|c|c|c|}
\hline 2 & 3 & 3 & 3 & 4 & 4 & 3. \\
\hline 2 & 2 & 3 & 3 & 4 & 4 & 2. \\
\hline 2 & 2 & 3 & 3 & 3 & 4 & 2. \\
\hline 2 & 2 & 3 & 3 & 3 & 4 & 1. \\
\hline 2 & 2 & 2 & 3 & 3 & 3 & 1. \\
\hline
\end{tabular}

\begin{tabular}{|c|c|c|c|c|c|c|}
\hline 2 & 2 & 3 & 3 & 3 & 4 & 3.0 \\
\hline 2 & 2 & 2 & 3 & 3 & 4 & 2.5 \\
\hline 2 & 2 & 2 & 3 & 3 & 3 & 2.0 \\
\hline 1 & 2 & 2 & 2 & 3 & 3 & 1.5 \\
\hline 1 & 1 & 2 & 2 & 3 & 3 & 1.0 \\
\hline 70 & 80 & 90 & 100 & 110 & 120 & \\
\hline
\end{tabular}

Glucose $=7.0 \mathrm{mmol} / \mathrm{l}$
Fig. 1 Nomogram illustrating how strata of the metabolic syndrome measures correspond to one standardised unit change in the continuous metabolic syndrome score (e.g. a score of 3 represents a change of $3 \mathrm{SD}$ in the score). The odds of increasing or decreasing associated metabolic syndrome outcomes per 1-unit (SD) change in the score over 9 years for our population are listed in Table 3 for diabetes and in Table 4 for coronary heart disease and cardiovascular disease; a clinical example is presented in the results. Trigly, triglycerides; $S B P$, systolic blood pressure 
matrix was analysed. Fasting insulin and triglycerides levels were $\log$-transformed to reduce skewness of distribution. Because the goal was to develop a simple, clinically useful nomogram from the principal components analysis, we also evaluated a simpler model without fasting insulin or HDL cholesterol, having previously confirmed a high correlation between the two scores for the syndrome and that prediction of diabetes and CVD outcomes was similar. Insulin was not included in the simpler model, as it is not routinely available in clinical practice. Triglycerides and HDL cholesterol are highly correlated with each other, and triglycerides were more highly correlated than HDLcholesterol with the score from the principal components analysis that included all six measures. For this reason, triglycerides were chosen to represent the lipid parameters. We identified the first principal component as a metabolic syndrome score. The coefficients of the metabolic syndrome measures in the score are the correlations between the measures and the first principal component. To retain the desirable property (for our study aim) that this score maximally spreads out the subjects, we did not further rotate the principal components.

After excluding subjects with diabetes at the baseline exam, we used logistic regression to predict incident diabetes among the 3,774 adults in whom glycaemic status had been determined after 9 years of follow-up. Similarly, we predicted incident CHD and CVD events among 3,910 subjects present at baseline. Standardised odds ratios (ORs) were used to describe incident diabetes, CHD, and CVD per 1-SD increase in the continuous metabolic syndrome score. The National Cholesterol Education Program (NCEP) definition of metabolic syndrome and its parameters are dichotomous, so to provide a comparison with NCEP-defined metabolic syndrome, we also evaluated the risk of incident diabetes, CHD, and CVD for an increase in one among the five possible dichotomised abnormalities as defined by NCEP [6]. We tested quadratic models for all predictors to confirm the relationships were linear for the final models. The Hosmer-Lemeshow goodness-of-fit test was used with each model [21], and all models provided a good fit.

To illustrate how such a continuous metabolic syndrome score could be used in practice, we created a nomogram from our data (Fig. 1), whereby the combined effect of different levels of these four syndrome measures (waist circumference, triglycerides, glucose, and systolic blood pressure) of the metabolic syndrome score can be determined. The scale of this nomogram corresponds to a 1-SD increase in the metabolic syndrome score, and this score is the weighted sum of the four standardised measures (Table 1), weighted by the first principal component coefficients (Table 2), men and women combined.

\section{Results}

Baseline characteristics of the 5,024 participants are shown in Table 1. Despite differences between men and women in body size and means of metabolic syndrome measures

Table 1 Baseline characteristics of the 5,024 participants in the D.E.S.I.R. cohort by sex

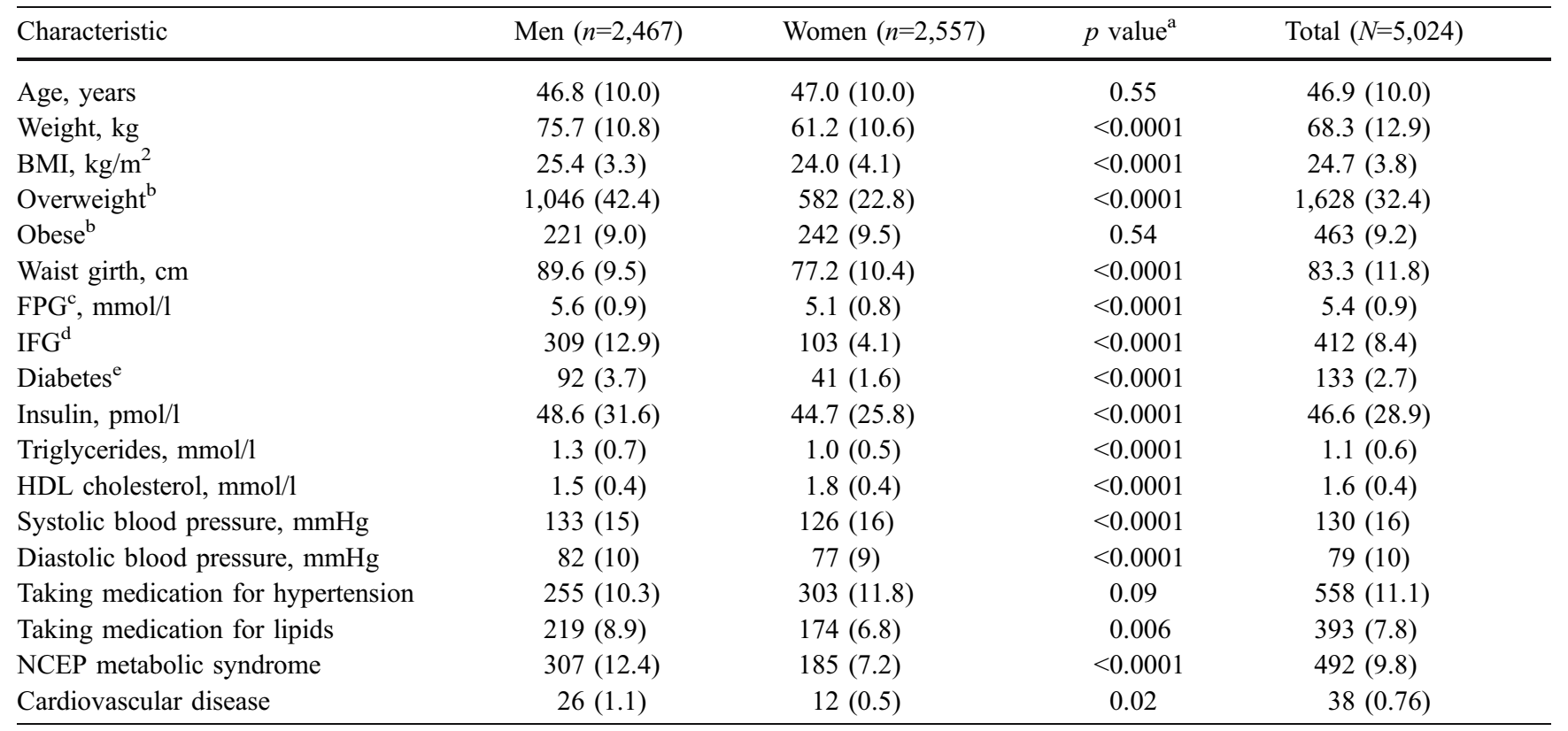

Data are presented as mean (SD) or $n(\%)$ for proportions

${ }^{a}$ Reported $p$ values comparing men and women were calculated by $t$-tests and $\chi^{2}$ comparisons for group means and proportions, respectively, with the Fisher exact test where necessary

${ }^{\mathrm{b}}$ Overweight=BMI $25-29.99 \mathrm{~kg} / \mathrm{m}^{2}$; obese=BMI $\geq 30 \mathrm{~kg} / \mathrm{m}^{2}$

${ }^{\mathrm{c}} F P G$ Fasting plasma glucose

${ }^{\mathrm{d}} \mathrm{IFG}=\mathrm{FPG}$ 6.10-6.99 $\mathrm{mmol} / 1$

${ }^{\mathrm{e}}$ Diabetes $=\mathrm{FPG} \geq 7.0 \mathrm{mmol} / 1$ or hypoglycaemic drug treatment 
Table 2 Percentages of variance explained and eigenvalues from the principal components analyses and the coefficients ${ }^{\mathrm{a}}$ of the measures in the metabolic syndrome, with six and four syndrome measures, defined from the first principal component ${ }^{\mathrm{b}}$

\begin{tabular}{|c|c|c|c|c|c|}
\hline & \multicolumn{2}{|c|}{ First principal component with six measures } & \multicolumn{3}{|c|}{$\begin{array}{l}\text { First principal component with four measures: } \\
\text { the metabolic syndrome score }\end{array}$} \\
\hline & Men $(n=2,467)$ & Women $(n=2,557)$ & Men $(n=2,467)$ & Women $(n=2,557)$ & Combined $(n=5,024)$ \\
\hline Percentage of variance explained & 41 & 40 & 45 & 46 & 50 \\
\hline Eigenvalue & 2.47 & 2.41 & 1.82 & 1.84 & 2.00 \\
\hline Waist circumference & 0.50 & 0.51 & 0.60 & 0.59 & 0.59 \\
\hline [Log] triglycerides & 0.46 & 0.45 & 0.50 & 0.52 & 0.51 \\
\hline Systolic blood pressure & 0.30 & 0.33 & 0.47 & 0.48 & 0.47 \\
\hline Glucose & 0.30 & 0.30 & 0.41 & 0.38 & 0.42 \\
\hline HDL cholesterol & -0.34 & -0.33 & & & \\
\hline$[\log ]$ insulin & 0.49 & 0.48 & & & \\
\hline
\end{tabular}

${ }^{\text {a }}$ The coefficients are the correlation coefficients between the measures and the first principal component, and they are also the coefficients of the standardised measures in the metabolic syndrome score

${ }^{\mathrm{b}}$ The mean and SD for each variable used in this first principal component are listed in Table 1, except for [log] triglycerides (mean=-0.03, $\mathrm{SD}=0.52$ ) and $[\mathrm{log}]$ insulin (mean $=0.43, \mathrm{SD}=0.03$ ) for men and women combined

(Table 1), the coefficients of the measures in the separate principal components analyses were remarkably similar (Table 2). Furthermore, the standard deviations of the overall metabolic syndrome scores were identical in both sexes (data not shown). We therefore used a common metabolic syndrome score for the entire population of men and women for our final analyses (Table 2). We also did separate analyses, excluding subjects treated with medication for hypertension, hyperlipidaemia and diabetes, and found the coefficients were very similar to those that included the treated population (data not shown). Thus, we used the full population (treated and untreated) to develop the common score.

The metabolic syndrome score, defined from the simpler analysis with waist circumference, fasting triglycerides, fasting glucose, and systolic blood pressure, was highly correlated with that from the principal components analysis that also included fasting insulin and HDL cholesterol $\left(r_{s}=0.94\right)$. Furthermore, the variable coefficients were similarly ranked, with waist circumference having the highest coefficient in both analyses (Table 2). Therefore, we chose the simpler model with the four metabolic syndrome measures. This metabolic syndrome score explained $50 \%$ of the total variance among these measures, and the measure coefficients (which are the correlations with the metabolic syndrome score) were: waist circumference $(0.59)$, [log]triglycerides $(0.51)$, systolic blood pressure (0.47), and glucose (0.42). Consistent with the notion of a syndrome with highly correlated measures, all metabolic syndrome correlation coefficients were above 0.40 and the eigenvalues for the first and second principal components, respectively, were 2.0 and 0.80 .

To confirm that this continuous metabolic syndrome score would be clinically meaningful, we used it to predict incident diabetes and CVD.

After 9 years of follow-up, $173(5 \%)$ of 3,774 participants became diabetic (122 men and 51 women). For every 1-SD increase in the continuous metabolic syndrome score, the age-adjusted standardised OR of incident diabetes significantly increased more than threefold in men and more than five-fold in women (Table 3). The ORs were higher than for an increase in one NCEP

Table 3 Age-adjusted ORs with 95\% CIs for risk of incident diabetes associated with the metabolic syndrome, among 3,774 D.E.S.I.R. participants followed for 9 years

\begin{tabular}{|c|c|c|c|c|}
\hline & \multicolumn{4}{|l|}{ Age-adjusted $\mathrm{OR}^{\mathrm{a}}(95 \% \mathrm{CI})$} \\
\hline & \multicolumn{2}{|l|}{ Men } & \multicolumn{2}{|l|}{ Women } \\
\hline & $\begin{array}{l}\text { Including } \text { IFG }^{\mathrm{b}} \text { at baseline } \\
(n=1,835)\end{array}$ & $\begin{array}{l}\text { Excluding IFG at baseline } \\
(n=1,599)\end{array}$ & $\begin{array}{l}\text { Including IFG at baseline } \\
(n=1,939)\end{array}$ & $\begin{array}{l}\text { Excluding IFG at baseline } \\
(n=1,868)\end{array}$ \\
\hline Cases of diabetes & 122 & 53 & 51 & 25 \\
\hline MetS score ${ }^{c}$ & $3.4(2.6-4.4)$ & $3.1(2.2-4.4)$ & $5.1(3.6-7.2)$ & $4.1(2.6-6.4)$ \\
\hline
\end{tabular}

MetS Metabolic syndrome

${ }^{a}$ OR for the MetS score is per 1-SD increase in the continuous MetS score; OR for MetS-NCEP is per increase of one NCEP abnormality, with a range of $0-5$ potential abnormalities as defined by NCEP [6]

${ }^{\mathrm{b}} \mathrm{IFG}=6.1-6.99 \mathrm{mmol} / \mathrm{l}$

${ }^{c}$ MetS score, defined from the first principal components analysis with four measures included: waist circumference, [log] triglycerides, glucose and systolic blood pressure 
abnormality. We did a separate analysis of incident diabetes using the metabolic syndrome score that also included insulin and HDL cholesterol. We found the OR of incident diabetes was no greater than for the simpler score (data not shown). After excluding subjects with IFG at baseline, the continuous metabolic syndrome score remained highly predictive of incident diabetes (Table 3). Results were similar even when subjects with the wider definition of IFG (fasting plasma glucose: $5.6-6.99 \mathrm{mmol} / 1[22,23]$ ) were excluded (data not shown).

Over the same 9-year follow-up, 77 subjects developed CHD (33 had a myocardial infarction, 29 a revascularisation, and 15 documented coronary disease); in addition, 16 had a stroke and four developed peripheral vascular disease, raising the total to 97 subjects with CVD. For men and women, respectively, the age-adjusted ORs for CHD were 1.8 (95\% CI 1.4-2.3) and 1.4 (0.78-2.6) and for CVD 1.7 (1.4-2.1) and 1.7 (1.0-2.7); they were more significant in men because women had only 14 incident CVD events (Table 4). Moreover, we also did separate analyses for the cardiovascular endpoints, excluding persons who had diabetes at baseline or who developed diabetes over the 9 years of follow-up. The ORs for CVD were similar in these analyses: $1.7(1.2-2.3)$ and $1.5(0.7-$ 2.8) for men and women, respectively; thus the increased risk of CVD is not due solely to the risk associated with diabetes. However, for women, the metabolic syndrome was no longer significant due to the small number of cases and hence low statistical power. Finally, we adjusted for the classical CVD risk factors, smoking and non-HDL cholesterol, which are not included in the metabolic syndrome. The ORs for CVD were slightly attenuated: $1.6(1.2-2.0)$ for men and $1.5(0.9-2.6)$ for women.

Although the metabolic syndrome score was strongly and linearly associated with the risk of incident diabetes, coronary, and cardiovascular disease, we also recognise that it is not practical for most clinicians to calculate such a score for individual patients. A nomogram using strata of the four syndrome measures used in this metabolic

Table 4 Age-adjusted ORs with 95\% CI for risk of incident CHD and CVD with the metabolic syndrome assessed among 3,910 D.E.S.I.R. participants followed for 9 years

\begin{tabular}{lll}
\hline & \multicolumn{2}{l}{ Age-adjusted OR OR $^{\mathrm{a}}(95 \% \mathrm{CI})$} \\
\cline { 2 - 3 } & \multicolumn{1}{l}{ Men $(n=1,919)$} & Women $(n=1,991)$ \\
\hline Cases of coronary heart disease & 68 & 9 \\
Metabolic syndrome score $^{\mathrm{b}}$ & $1.8(1.4-2.3)$ & $1.4(0.78-2.6)$ \\
Metabolic syndrome-NCEP & $1.5(1.2-1.8)$ & $1.5(0.94-2.5)$ \\
Cases of cardiovascular disease & 83 & 14 \\
Metabolic syndrome score $^{\mathrm{b}}$ & $1.7(1.4-2.1)$ & $1.7(1.0-2.7)$ \\
Metabolic syndrome-NCEP & $1.4(1.2-1.8)$ & $1.7(1.1-2.5)$ \\
\hline
\end{tabular}

${ }^{\mathrm{a}} \mathrm{OR}$ for the metabolic syndrome score is per 1-SD increase in the continuous metabolic syndrome score; OR for metabolic syndromeNCEP is per increase of one NCEP abnormality, with a range of 0 5 potential abnormalities as defined by NCEP [6]

${ }^{b}$ Metabolic syndrome score, defined from the first principal components analysis with four measures included: waist circumference, $[\log ]$ triglycerides, glucose and systolic blood pressure syndrome score illustrates how this continuous metabolic syndrome score could be translated into a useful clinical tool (Fig. 1) to evaluate both the degree of severity of the metabolic syndrome, as well as the change in risk of metabolic syndrome outcomes, such as diabetes and CHD, that is associated with a 1-unit (SD) change in the metabolic syndrome score. The corresponding mean metabolic syndrome score for our population was 2.0 for men and 1.1 for women. As an example of how such a nomogram could be used in a busy practice to counsel individual patients, a man with an initial glucose of $5.6 \mathrm{mmol} / 1$, systolic blood pressure of $160 \mathrm{mmHg}$, a waist circumference of $110 \mathrm{~cm}$, and triglycerides of $2.5 \mathrm{mmol} / \mathrm{l}$ would have a score of ' 4 ' on the nomogram. If his waist decreases to $100 \mathrm{~cm}$ (all other parameters unchanged), his score on the nomogram will decrease by 1 SD to ' 3 ', and thus he would have a 3.4-fold lower risk of incident diabetes and a 1.8-fold decreased risk of incident CHD over a 9-year period (see Tables 3 and 4 for OR per SD change in the metabolic syndrome score). If, at the same time that his waist circumference decreases to $100 \mathrm{~cm}$, his triglycerides also decrease to $1.5 \mathrm{mmol} / \mathrm{l}$ and his systolic blood pressure to $140 \mathrm{mmHg}$, his score on the nomogram will be ' 2 ', a decrease of 2 SD for the metabolic syndrome score, and his odds of incident diabetes will now be decreased by $(3.4)^{2}=12$, and his odds of incident CHD will be reduced by $(1.8)^{2}=3.2$ over a 9 -year period.

\section{Discussion}

In this population of 5,024 middle-aged adults, we found that the continuous metabolic syndrome score, defined by the first principal component determined from principal components analysis, explained $50 \%$ of the variance among the selected metabolic syndrome measures (waist circumference, glucose, triglycerides, and systolic blood pressure). As might be expected for a syndrome, all of these metabolic syndrome measures were highly correlated with the metabolic syndrome score. Interestingly, the waist circumference had the highest correlation coefficient or "weight" of all measures with the metabolic syndrome score, and when insulin was included in the six-variable principal components analysis, the correlations between the first principal component and insulin and waist circumference were almost identical.

The metabolic syndrome is a known marker of risk for both incident diabetes and cardiovascular disease and this metabolic syndrome score predicted both. However, if an individual's glucose is already elevated (e.g. IFG), then he or she is likely to already be on the pathway to developing diabetes $[22,23]$. Therefore, the utility of the metabolic syndrome in predicting diabetes is greater, if it can identify people at high risk of incident diabetes in those whose blood glucose concentrations are still normal. Importantly, our continuous metabolic syndrome score was still significantly associated with more than a three-fold, ageadjusted increased OR of incident diabetes in both men and women, even when those with IFG at the baseline exam 
were excluded. Further, the metabolic syndrome score predicted CVD in men who did not become diabetic over the follow-up, thus indicating that the effect of the syndrome is not just diabetes-related; for women, the OR for the syndrome was lower and no longer significant. The ORs for CVD were attenuated when adjustment was made for the additional classic risk factors, smoking and nonHDL cholesterol.

One of the strengths of our study is that it comes from a large prospective cohort of community-dwelling, middleaged adults who are not patients, and is a study that was specifically designed to evaluate the metabolic syndrome and its associated outcomes by a standardised protocol.

A potential limitation of our study is that not all participants present at the baseline exam attended subsequent examinations. However, comparing those lost to follow-up with those followed for incident outcomes, we found no significant difference between baseline mean fasting glucose concentrations $(5.3 \mathrm{mmol} / \mathrm{l}$ for both). Other differences among metabolic syndrome measures revealed slightly worsened metabolic syndrome baseline measures in those lost to follow-up (e.g. baseline waist circumference was $84 \mathrm{vs} 83 \mathrm{~cm}$ in those absent vs present at the year 9 exam, $p=0.007$ ). Thus, if anything, losses to follow-up may attenuate the relationship between metabolic syndrome and both incident diabetes and CVD.

The current dichotomised definitions of the metabolic syndrome, and the NCEP ones in particular, were developed by expert committees as a clinically useful means of evaluating the metabolic syndrome with an individual patient [4-8]. However, by dichotomising the individual measures, information is lost. For example, a very minor improvement in one variable could result in an individual no longer being classified as having the syndrome, despite minimal change in presumed risk. This issue is not trivial, as one-third of those in our cohort who were initially classified as having the NCEP metabolic syndrome were re-classified as 'normal' by the same criteria after a 3-year follow-up, despite minimal changes in absolute values of measures for many participants [11]. By evaluating the syndrome on the basis of a continuous score, a modest change in only one variable will result in only a modest change in the overall metabolic syndrome score.

Our analyses demonstrated that the risk associated with the syndrome appears to be linear, even among people with normal glucose. Furthermore, the "weight" of each of the measures is not equal, with waist circumference having the highest "weight," being the most correlated with our metabolic syndrome score (Table 2).

The challenge, then, is how to devise a practical, quantitative variable that combines the data available from each of the individual measures so that the syndrome can be evaluated on the basis of a continuous score. A syndrome exists because of the correlations between the measures, and a syndrome definition should be based on this information.

Factor analysis has been used extensively to describe the metabolic syndrome [14-20] and the factors have been shown to predict diabetes and CVD [17-20]. It is useful for identifying new unknown or latent factors, to determine the structure of the data, in this case the structure of the syndrome. Usually, only those factors that explain most of the variance are kept, and they are then rotated so that each factor is correlated with only two or three of the original measures [12, 13]. However, by design, this rotation precludes the retention of the unrotated first factor, which describes what all measures have in common.

In contrast, principal components analysis (without rotation) aims to find the best linear combinations of all the measures and the first principal component, by definition, has the maximum variance. It is an appropriate mathematical technique to determine a continuous syndrome score. The first principal component was indeed well correlated with all of the syndrome measures (correlation coefficients $0.30-0.60$, Table 2). Further, the second principal component had an eigenvalue of only 0.8 , indicating that only the first principal component provided more information than any one of the individual metabolic syndrome measures included in the analysis. This continuous metabolic syndrome score predicted incident diabetes, coronary, and cardiovascular disease.

We are aware of only four studies of the metabolic syndrome that have aimed to find a quantitative evaluation of the syndrome using a similar approach, all of which evaluated the principal components after rotation [17-20]. To our knowledge, our study is the first to use principal components analysis with the aim of defining the syndrome on the basis of a continuous score.

Despite the differences between men and women in our study, in both the average metabolic syndrome score and its associated risks per SD increase (Tables 3,4), a common nomogram of the metabolic syndrome score was possible (Fig. 1). The prevalence of diabetes observed by us is lower than in other European countries, and there was a nearly two-fold higher prevalence of diabetes (defined on fasting glucose and treatment) in men than in women [24]. This increased male:female ratio of diabetes prevalence was also seen in an American population 25 years ago, when men were on average slightly more overweight than women [25]. CVD is less frequent in France than in many other countries [26]. However, using the number of NCEP abnormalities as a continuous function, the ORs of 1.4 and 1.7 that we observed for CVD events in men and women, respectively, were similar to the 1.29 from the Hoorn study for both men and women, and similar to that for cardiovascular mortality in the US MRFIT study of $>10,000$ men, $1.25[27,28]$. Thus, it is possible that the CVD risk associated with the metabolic syndrome abnormalities is similar in France to that in other countries, despite a lower overall CVD incidence. However, validation of our continuous metabolic syndrome score in other populations, particularly more obese populations, will be necessary, as well as testing of its capacity to predict diabetes and CVD.

Among a community-dwelling population of middleaged adults, recruited with the aim of studying the metabolic syndrome, we found that the first principal component from a principal components analysis provided 
a metabolic syndrome score which was highly correlated with all of the syndrome measures. Furthermore, we found that the metabolic syndrome score predicted incident diabetes and cardiovascular disease. Our results, as illustrated with a nomogram, suggest that it is possible to evaluate the severity of the metabolic syndrome as a continuous variable, using its measures continuously, rather than dichotomised with arbitrary cut-points [4-8]. Moreover, our results provide an initial step to meeting the ADA-EASD urgent call for research on new ways of evaluating the metabolic syndrome and risk of its associated outcomes [10].

Acknowledgements T. A. Hillier was supported by an ADA-EASD Trans-Atlantic Fellowship.

This work was also supported by the National Health and Medical Research Institute (INSERM), National Health Assurance for the Workforce (CNAMTS), the French Cardiology Federation, the French Foundation, the French Speaking Association for the Study of Diabetes and Metabolic Disorders (ALFEDIAM), the National Interprofessionals' Office for Wine (ONIVINS), the Diabetes Vascular Risk Association, the Inter Regional Health Institute (IRSA), Ardix Medical, Bayer Diagnostics, Becton Dickinson, Cardionics, Lipha Pharmaceuticals, Merck Santé, Novo Nordisk, Pierre Fabre, and Topcon.

The D.E.S.I.R. Study Group: INSERM U258: B. Balkau, P. Ducimetière, E. Eschwège; INSERM U367: F. Alhenc-Gelas; CHU d'Angers: Y. Gallois, A. Girault; Hôpital Bichat: M. Marre; Health Examination Centres of region 9: Alençon, Angers, Blois, Caen, Chartres, Châteauroux, Cholet, Le Mans, Orléans, Tours; General Practice Research Institute: J. Cogneau; General Practitioners in the French Departements; Inter Regional Health Institute: C. Born, E. Cacès, M. Cailleau, J. G. Moreau, F. Rakotozafy, J. Tichet, S. Vol. We also thank M. Sucec for her editorial assistance.

Duality of interest The authors declare that they have no duality of interest.

\section{References}

1. Reaven GM (1988) Banting lecture. Role of insulin resistance in human disease. Diabetes 37:1595-1607

2. DeFronzo RA, Ferrannini E (1991) Insulin resistance. A multifaceted syndrome responsible for NIDDM, obesity, hypertension, dyslipidemia, and atherosclerotic cardiovascular disease. Diabetes Care 14:173-194

3. Stern MP (1995) Diabetes and cardiovascular disease. The "common soil" hypothesis. Diabetes 44:369-374

4. World Health Organization Consultation (1999) Part 1: Diagnosis and classification of diabetes mellitus. In: Report of a WHO Consultation (eds) Definition, diagnosis and classification of diabetes mellitus and its complications. WHO, Geneva, Switzerland

5. Balkau B, Charles MA (1999) Comment on the provisional report from the WHO consultation. European Group for the Study of Insulin Resistance (EGIR). Diabet Med 16:442-443

6. Expert Panel on detection, evaluation, and treatment of high blood cholesterol in adults (2001) Executive summary of the third report of The National Cholesterol Education Program (NCEP) expert panel on detection, evaluation, and treatment of high blood cholesterol in adults (Adult Treatment Panel III). JAMA 285:2486-2497

7. Alberti KG, Zimmet P, Shaw J; IDF Epidemiology Task Force Consensus Group (2005) The metabolic syndrome - a new worldwide definition. Lancet 366:1059-1062
8. Grundy SM, Cleeman JI, Daniels SR et al (2005) Diagnosis and management of the metabolic syndrome: an American Heart Association/National Heart, Lung, and Blood Institute scientific statement. Circulation 112:2735-2752

9. Ford ES (2005) Risks for all-cause mortality, cardiovascular disease, and diabetes associated with the metabolic syndrome: a summary of the evidence. Diabetes Care 28:1769-1778

10. Kahn R, Buse J, Ferrannini E, Stern M (2005) The metabolic syndrome: time for a critical appraisal. Joint statement from the American Diabetes Association and the European Association for the Study of Diabetes. Diabetologia 48:1684-1699

11. Balkau B, Vernay M, Mhamdi L et al (2003) D.E.S.I.R. Study Group. The incidence and persistence of the NCEP (National Cholesterol Education Program) metabolic syndrome. The French D.E.S.I.R. study. Diabetes Metab 29:526-532

12. Jackson JE (1991) A user's guide to principal components analysis. Wiley, New York

13. Jolliffe IT (2002) Principal components analysis, 2nd edn. Springer, Berlin Heidelberg New York

14. Meigs JB (2000) Invited commentary: insulin resistance syndrome? Syndrome X? Multiple metabolic syndrome? A syndrome at all? Factor analysis reveals patterns in the fabric of correlated metabolic risk factors. Am J Epidemiol 152:908-911

15. Lawlor DA, Ebrahim S, May M, Davey Smith G (2004) (Mis) use of factor analysis in the study of insulin resistance syndrome. Am J Epidemiol 159:1013-1018

16. Maison P, Byrne CD, Hales CN et al (2001) Do different dimensions of the metabolic syndrome change together over time? Evidence supporting obesity as the central feature. Diabetes Care. 24:1758-1763

17. Hanson RL, Imperatore G, Bennett PH et al (2002) Components of the 'metabolic syndrome' and incidence of type 2 diabetes. Diabetes 51:3120-3127

18. Kekalainen P, Sarlund H, Pyorala K, Laakso M (1999) Hyperinsulinemia cluster predicts the development of type 2 diabetes independently of family history of diabetes. Diabetes Care 22:86-92

19. Lempiainen P, Mykkanen L, Pyorala K, Laakso M, Kuusisto J (1999) Insulin resistance syndrome predicts coronary heart disease events in elderly nondiabetic men. Circulation 100:123-128

20. Tang W, Miller MB, Rich SS et al (2003) National Heart, Lung, and Blood Institute Family Heart Study. Linkage analysis of a composite factor for the multiple metabolic syndrome: the National Heart, Lung, and Blood Institute Family Heart Study. Diabetes 52:2840-2847

21. Hosmer DW, Lemeshow S (1989) Assessing the fit of the model. Applied logistic regression. Wiley, New York

22. American Diabetes Association (2004) Diagnosis and classification of diabetes mellitus. Diabetes Care 27:S5-S10

23. Genuth S, Alberti KG, Bennett P et al (2003) Expert Committee on the Diagnosis and Classification of Diabetes Mellitus. Follow-up report on the diagnosis of diabetes mellitus. Diabetes Care 26:3160-3167

24. DECODE Study Group (2003) Age- and sex-specific prevalences of diabetes and impaired glucose regulation in 13 European cohorts. Diabetes Care 26:61-69

25. Barrett-Connor E (1980) The prevalence of diabetes mellitus in an adult community as determined by history or fasting hyperglycemia. Am J Epidemiol 111:705-712

26. Kuulasmaa K, Tunstall-Pedoe H, Dobson A et al (2000) Estimation of contribution of changes in classic risk factors to trends in coronary-event rates across the WHO MONICA Project populations. Lancet 355:675-687

27. Dekker JM, Girman C, Rhodes T et al (2005) Metabolic syndrome and 10-year cardiovascular disease risk in the Hoorn Study. Circulation 112:666-673

28. Eberly LE, Prineas R, Cohen JD et al (2006) Metabolic syndrome: risk factor distribution and 18-year mortality in the multiple risk factor intervention trial. Diabetes Care 29:123-130 